\title{
Germanica
}

$47 \mid 2010$

« Krack ! Tschock ! Pflatsch ! Bummmm ! La BD de langue allemande (à suivre...) »

\section{BD et dictature : Vater und Sohn, soumission à la censure ou révolte discrète?}

Vater und Sohn und die Diktatur. Unterwerfung oder heimliche Rebellion?

The Graphic Novel und Dictatorship. Was Father and Son submissive to

cesureship, or subtley subversive?

\section{Sylvain Farge}

\section{(2) OpenEdition}

Journals

Édition électronique

URL : http://journals.openedition.org/germanica/1104

DOI : $10.4000 /$ germanica. 1104

ISSN : 2107-0784

Éditeur

Université de Lille

Édition imprimée

Date de publication : 31 décembre 2010

Pagination : $37-50$

ISBN : 9782913857261

ISSN : 0984-2632

Référence électronique

Sylvain Farge, «BD et dictature : Vater und Sohn, soumission à la censure ou révolte discrète ?», Germanica [En ligne], 47 | 2010, mis en ligne le 01 décembre 2012, consulté le 06 octobre 2020. URL: http://journals.openedition.org/germanica/1104; DOI : https://doi.org/10.4000/germanica.1104

Ce document a été généré automatiquement le 6 octobre 2020.

(c) Tous droits réservés 


\title{
BD et dictature : Vater und Sohn, soumission à la censure ou révolte discrète?
}

\author{
Vater und Sohn und die Diktatur. Unterwerfung oder heimliche Rebellion? \\ The Graphic Novel und Dictatorship. Was Father and Son submissive to \\ cesureship, or subtley subversive?
}

\section{Sylvain Farge}

„Selten erschienen Werke, die die Leser als verschlüsselte Regimekritik verstehen mochten,

gleich ob sie als solche intendiert waren"

(Möller, 1984, 33) ${ }^{1}$

1 La bande dessinée Vater und Sohn, constituée non pas d'albums d'aventures mais d'une série de strips autonomes n'excédant pas six vignettes, parue de manière hebdomadaire entre 1934 et 1936, a connu une prospérité indéniable. Cela tient probablement à son humour a priori affable et à la grande humanité des deux héros. Sans compter que les strips sont souvent sans légende et les histoires relativement aisées à décrire, ce qui en a fait un outil pédagogique de choix dans l'enseignement de l'allemand langue étrangère. À tel point que l'on oublie aisément que la BD est parue sous le Troisième Reich et la censure. Quand on le reconnaît, c'est souvent pour balayer toute dimension critique et argumenter que l'auteur s'est réfugié dans une innocente idylle sans prétention critique (F. Klinkers, 1976). N'est-ce pas faire un procès rapide à une œuvre plus complexe qu'il n'y paraît au premier abord? La forme même de cette œuvre, la BD, souvent attachée dans les représentations à la culture enfantine (voire infantile), a sûrement participé à ce jugement.

2 Nous nous proposons d'analyser la bande sous un angle neuf pour voir si ne se cache pas sous les dehors primesautiers une œuvre plus grave, les monstres des profondeurs, comme les a nommés Gerd Ueding en référence à Shakespeare (2007). Nous montrerons, dans un premier temps, que l'auteur, dans la limite des contraintes 
imposées, a fait preuve de plus de courage et d'inventivité qu'on ne lui en a souvent prêtés. Dans un deuxième temps, nous verrons comment il a exploité les ressources que lui offrait précisément le medium BD pour s'exprimer avec une liberté de ton et un courage qu'il serait temps de reconnaître.

\section{Origine et évolution d'une œuvre en clair-obscur}

3 La bande dessinée Vater und Sohn a connu un grand succès lors de sa publication dans la Berliner Illustrirte. A priori, les aventures hebdomadaires d'un père bonhomme et ventripotent et de son fils espiègle sont une distraction bienvenue en ces premières années de dictature du Troisième Reich. Le dessinateur, e.o.plauen, est décidément très doué pour croquer des scènes anodines et pleines de tendresse entre un père et son fils. Et pourtant, e.o.plauen n'est jamais qu'Erich Ohser, connu sous la République de Weimar pour ses caricatures mordantes.

4 E. Ohser s'est fait alors un nom pour ses caricatures politiques, qui écorchent avec prédilection Josef Goebbels, l'homme d'affaire et politicien Alfred Hugenberg, ministre dans le premier gouvernement de Hitler, et le NSDAP. La caricature la plus connue est probablement celle dans laquelle un homme trace une croix gammée dans la neige avec un jet d'urine (Service à la nation, Dienst am Volk, Neue Revue, 1931). Sans être affilié à la social-démocratie, Ohser n'a pas caché ses sympathies et il les paiera d'ailleurs de sa vie en 1944. Il fait preuve d'un esprit caustique aussi bien dans ses caricatures politiques que sociales (e.g. Ça ne devrait pas être permis, Das dürfte nicht erlaubt sein, où une femme fait une pelote avec les boyaux d'un homme hurlant de douleur, dans Neue Revue, 1931). Le contraste est saisissant avec les histoires apparemment «innocentes et pures » du père et de son fils. Un caricaturiste sans concession peut-il subitement dessiner des planches d'une grande fraîcheur, qui rappellent la tradition des chenapans espiègles?

Il faut toutefois se rendre à l'évidence : le dessinateur s'est vu refuser le 27 janvier 1934 l'inscription au Landesverband Berlin der deutschen Presse (Organisme de presse de Berlin), ce qui était synonyme d'interdiction d'exercer. La raison en était ses dessins considérés comme d'inspiration marxiste, parus entre autres dans le journal Vorwärts (l'organe de presse de la social-démocratie d'alors, encore actuel). Un rédacteur de la maison d'édition Ullstein, ayant reconnu son talent, intercéda en sa faveur malgré son passé et les risques que cela comportait et obtint que ce dernier publie dans la Berliner Illustrirte, à condition toutefois qu'il se cantonne dans un dessin apolitique sous pseudonyme. Ohser décide alors de garder ses initiales e.o. et d'ajouter le nom de sa ville natale de Saxe, Plauen: e.o.plauen. Detlev Laubach $(2000,23)$ fait très judicieusement remarquer que, de la sorte, Ohser ne renie pas son identité: nous aimerions précisément montrer que l'auteur compose toujours au mieux avec les contraintes pour continuer à s'exprimer. Nous devrons pour cela nous contenter des dessins publiés, un grand nombre des esquisses et originaux ayant disparu lors du bombardement de Berlin de novembre 1943.

Des arguments forts parlent en faveur d'une BD au fond apolitique. Ainsi, les aventures du père et du fils relèvent très souvent de l'observation amusée d'un quotidien que tout un chacun pourrait vivre. Ainsi, dans la première bande, Le mauvais devoir à la maison (Der schlechte Hausaufsatz, 50/1934), le père, aide son fils à faire ses devoirs. Le lendemain, le fils est ramené à la maison par l'instituteur, qui le tire par l'oreille et administre une correction magistrale au père. On peut voir au mieux dans cette 
caricature une légère critique de l'autorité des instituteurs de l'époque, un rappel de la pédagogie wilhelminienne que vécut l'auteur ou une remise en cause de l'autorité et de la toute-puissance du père : ce motif est récurrent. Ainsi, dans Le bon exemple (Das Gute Beispiel, 10/1935), le père prend la place de son fils sur le fauteuil du dentiste pour lui donner l'exemple mais, quand le dentiste diagnostique une carie, le père fait le même caprice que son fils une minute auparavant. Dans une autre bande, Le goûter sacrifié (Das geopferte Frühstücksbrot, 1936), l'ambiance reste très amène, quoique avec une pointe d'amertume : l'enfant offre son encas aux moineaux quand arrive une femme riche qui les attire avec un plein cornet de nourriture pour le plaisir de se faire photographier par son accompagnateur. Le fils en pleure, mais se remet à offrir ses miettes aux moineaux après le départ du couple importun : un ange, qu'il ne voit pas, l'embrasse alors qu'il est occupé avec ses protégés (Cf. l'obole de la veuve, St Luc, 21, 1-4). La dimension morale n'est pas toujours aussi claire mais reste assez présente dans l'ensemble de l'œuvre, avec certains motifs récurrents, comme les contradictions dans l'éducation que le père donne à son fils.

7 Cette critique reste relativement innocente et n'a guère de portée politique réelle. De ce point de vue, l'œuvre semble souscrire à l'émigration intérieure : l'auteur se réfugie dans « l'idylle ou bien les rapports humains, réputés simples et atemporels, il fuit dans les valeurs traditionnelles et s'attache à mettre en scène le Vrai et l'Impérissable : [...] on fuit la trivialité et la barbarie du réel pour se réfugier dans le Beau, le Noble, l'Eternel » (Reichel, 1993 : 328 ; traduction personnelle²).

8 Vers la fin de 1935, la dimension critique et l'humour grinçant se font toutefois de plus en plus présents: les tours espiègles du fils font peu à peu place à des bandes plus sombres. Ainsi de la bande À la guerre, tous les coups sont permis (Im Krieg sind alle Mittel erlaubt, 44/1935). Père et fils jouent à la bataille navale dans la baignoire. Le père ayant touché l'un de ses bateaux, le fils se venge en douchant son père qui se penchait pour couler le navire touché. La réflexion sur la vengeance comme l'évocation de la guerre incitent à réfléchir et à interpréter la bande. Dans L'Intention était bonne (Gut gemeint, 27/1936), le père et le fils ont pêché un petit poisson. Au moment où son père veut le cuisiner, le fils se révolte tant que le père va le relâcher dans la rivière, où il se fait avaler par un poisson plus gros que lui... une note pessimiste qui contraste avec le ton bon enfant des bandes précédentes. Dans les bandes suivantes, ces sons discordants gagnent en importance.

9 Ainsi, dans La Surprise du lapin de Pâques (Überraschung für den Osterhasen, 15/1936), les deux héros volent les œufs du lapin de Pâques et peignent sur chacun d'entre eux la figure du père. Quand le lapin se retourne et voit tous ces regards fixés sur lui, il s'enfuie, effrayé. La tradition innocente et enfantine est ici détournée, la dimension du conte remise en cause. D'autres parodies suivent, comme Schnurps attrape la balle (Schnurps als Kugelfänger, 1936) : un renard a volé une oie (Allusion à la célèbre comptine Renard, tu as volé l'oie, Fuchs, du hast die Gans gestohlen). Le père tire sur le renard mais le chien Schnurps attrape la balle au vol et le renard s'enfuit avec l'oie dans la gueule. Dans Rencontre à la Pentecôte (Pfingstbekanntschaft, 1936), de même, le père et le fils voguent sur une barque quand apparaît une femme, à laquelle ils offrent le café. Le café $\mathrm{bu}$, les larmes aux yeux, elle montre sa queue de sirène et disparaît, laissant le père et le fils pleurer sa perte. Dans les caricatures suivantes, les allusions au monde enfantin se font plus rares mais le ton dysphorique persiste. 
10 Toujours en 1935, ainsi, deux strips publiés ailleurs que dans la Berliner Illustrirte marquent particulièrement clairement une vision relativement pessimiste. Dans L'Empreinte du temps (Vergänglichkeit, 40/1935), le père et le fils attrapent une grenouille et la mettent dans un bocal. Passe un héron qui l'avale et, prenant son envol, lâche une fiente, annonciatrice du devenir de la grenouille, dans le bocal. Dans Le Portrait (Porträtfotografie, 47/1935), facétieux, père et fils montrent leur postérieur au photographe : celui-ci, voyant la supercherie au tirage, peint des visages sur les fesses présentées et offre leur portrait au père et au fils. Dans ces cas, la critique ou le désespoir ne sont pas clairement annoncés, ils se lisent entre les lignes. Il faut donc replacer les bandes dans leur contexte et, allant au-delà du scénario, reconnaître l'humeur qu'elles véhiculent ou dont elles témoignent pour se convaincre que Vater und Sohn ne dépeint pas un monde idéal, atemporel et idyllique. L'irrévérence, l'ironie et la désinvolture, qui vont croissant avec le temps, incitent à voir dans ces saynètes apparemment légères une véritable prise de position philosophique, que la censure n'autorise pas à s'exprimer de manière trop univoque. Les Jeux Olympiques donneront à e.o.plauen l'occasion de poursuivre sa critique de manière plus explicite, quoique toujours prudente.

11 Dans la deuxième moitié de l'année 1936 (les jeux ont lieu du premier au 16 août 1936), ainsi, le thème du sport et de la force occupe le premier plan. Ainsi, dans La peur est le meilleur moteur (Angst macht Beine, 31/1936), le père dispute son fils, qui se tient sur la ligne de départ d'une course. Le fils, qui part pour échapper à l'ire paternelle au même moment que les coureurs, passe le premier la ligne d'arrivée. Retenons aussi Esprit contre Force (Geist gegen Kraft, 44/1936) : le père vient de planter un arbre quand arrive son fils, poursuivi par un homme aussi large et musclé que rustre et violent. Le fils se réfugie alors derrière son père, qui prend l'arbre juste planté d'une main et le déracine sans peine. Le poursuivant, incapable de réfléchir, prête au père une force surhumaine et part en courant. Quand on connaît la fascination des nazis pour la force, le sport comme éthique anti-intellectuelle de retour à la nature pure et non-corrompue, ces bandes, qui pourraient paraître autrement relativement anodines, peuvent tout à fait prendre une dimension politique et livrer une critique, certes discrète mais non moins réelle, du Troisième Reich et de l'invincibilité de l'athlète nazi.

Un peu plus tard, en mars 1937, la critique se fait à la fois plus osée et plus subtile, sous réserve d'interprétation. Dans L'Exemple à ne pas suivre (Warnendes Beispiel, 15/1936, paru dans l'anthologie de Ullstein), le père, voyant son fils jouer avec ses spaghettis, l'emmène au musée devant le groupe de Laocoon aux prises avec les serpents. Le gag paraît en soi innocent, mais il est aussi l'occasion de rappeler l'histoire du prêtre grec, démembré et tué avec ses fils par deux serpents entre autres pour avoir voulu mettre en garde les Troyens contre le Cheval de Troie. On peut aussi lire : qui fera connaitre sa crainte des nazis sera condamné. Une autre allusion est celle d'Un poisson rouge qui n'en était pas un (Abenteuer mit dem Goldfisch, 9/1937) : un marin offre un poisson rouge à l'enfant, qui le nourrit consciencieusement avec son père. Voilà toutefois que le poisson rouge, bientôt trop gros pour l'aquarium, doit migrer dans la baignoire, où il est nourri de seaux entiers de poissons... jusqu'au jour où, devenu baleine adulte, il fait exploser la maison. Ceci peut tout à fait être vu comme une métaphore de la montée du nazisme, qui, de petit parti sans importance, a crû jusqu'à détruire l'Allemagne. 

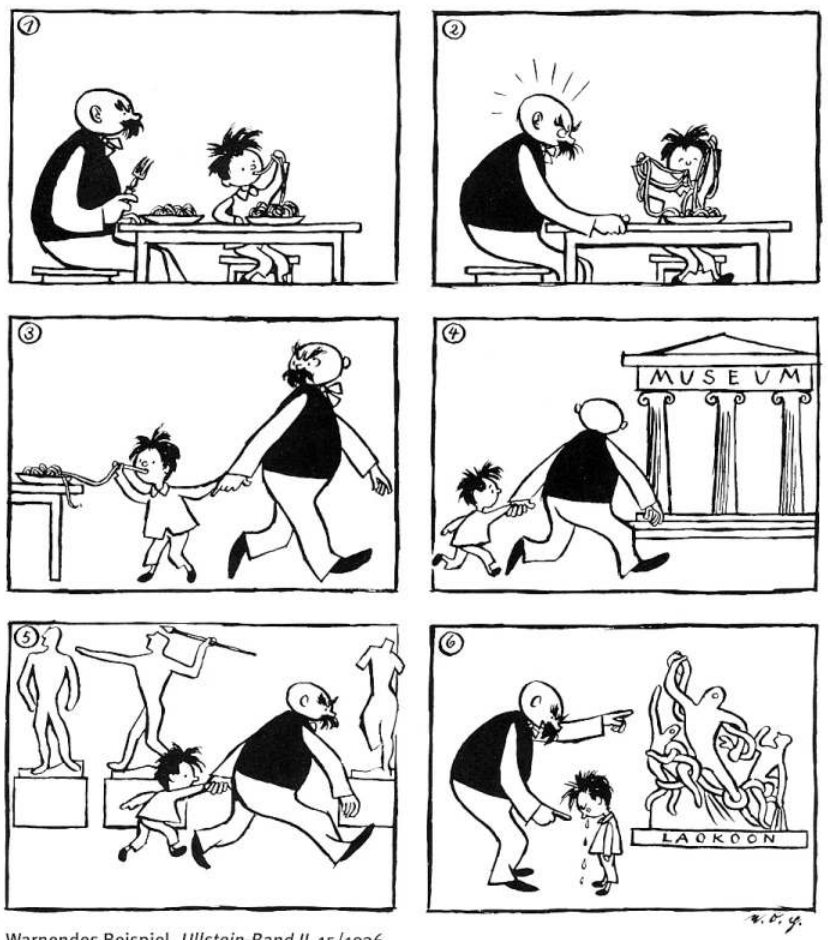

Warnendes Beispiel, Ullstein-Band II, 15/1936

Source : Ohser, E. (e.o.plauen), Gesamtausgabe, Politische Karikaturen, Zeichnungen, Illustrationen und alle Bildgeschichten Vater und Sohn, Contance, Südverlag GmbH, 2000.

Dès le gag suivant Un héritage miraculeux (Die große Erbschaft, 10/1937), les aventures de nos deux personnages prennent une nouvelle tournure: dans la première série ( $L a$ fortune, Im Reichtum), ils héritent une somme colossale et vivent en conséquence (mais sans finalement se déparer de leur caractère et de leurs comportements invétérés). Dans la seconde série (Seuls sur une île déserte, Auf einsamer Insel), partis en croisière, ils finissent naufragés (Incident pendant la croisière estivale, Zwischenfall auf der Sommerreise, 24/1937) et se mettent à vivre la vie des Robinson Crusoë. D'aucuns ont voulu voir dans ces deux séries le signe d'un essoufflement de l'auteur, qui aurait cherché un nouveau cadre pour trouver une nouvelle source d'inspiration. Le jugement est toutefois un peu rapide et, replacés dans leur contexte, ces changements ne sont plus aussi anodins. D'une part, en effet, l'héritage arrive juste la semaine suivant le gag de la baleine. Introduire la féérie à un moment où la vie devient toujours plus précaire en Allemagne pour certaines franges de la population n'est sûrement pas innocent pour un auteur qui évoque par métaphore son inquiétude devant la montée du nazisme. Au reste, l'humour doux-amer rappelle le ton caustique du caricaturiste de Weimar, pour qui la réalité n'est pas toujours aussi rose qu'il n'y paraît.

Ainsi, l'argent ne fait pas le bonheur : faute de savoir décortiquer le homard, père et fils quittent le château en colère et se retrouvent autour d'une simple saucisse dans la rue, comme au bon vieux temps (Marre du homard, Kummer mit Hummer, 13/1937). Plus tard, dans Chacun à sa place (Das schickt sich nicht, 18/1937), le fils voudrait jouer avec les enfants dans la rue. Impressionné par le regard réprobateur de ses chauffeurs, cependant, le père fait remonter son fils. La nuit venue, tous deux ne rêvent que de ce moment de bonheur perdu. L'humour au fond assez acide de l'auteur se retrouve tout particulièrement dans un gag de cette série, Le fantôme du manoir (Das Schlossgespenst, 16/1937) : le fantôme, avec une tête ronde vissée sur le corps textile, tire père et fils de 
leur sommeil. Le fils, ravi, joue au ballon avec la tête du fantôme, qu'il envoie rouler sous une commode. Vexé et en colère, le fantôme repart. Cet esprit noir dénote une fois de plus une certaine position face à la vie, qui n'est pas nécessairement celle, harmonieuse et reposante, que l'on prête traditionnellement à l'émigration intérieure. L'auteur donne forme à son pessimisme sous une forme aussi édulcorée que possible, si bien que c'est le style qui est porteur de ses inquiétudes, plus que le sujet lui-même. À ne voir que l'argument de la relation complice entre père et fils, on risque de ne pas percevoir la dysphorie, la peur, l'angoisse.

Le même clair-obscur inquiétant accompagne nos héros naufragés dans la seconde série: tout d'abord, comment en arrivent-ils là (Incident pendant la croisière estivale, Zwischenfall auf der Sommerreise, 24/1937) ? Accoudés au bastingage, ils voient une main, un doigt pointé vers le ciel, et se jettent à l'eau au secours du pauvre malheureux, qui se révèle être un panneau directionnel taillé en main... trop tard, le paquebot est loin (Cf. aussi La Gratitude, Der Dank, 28/1937). Vision assez pessimiste du geste altruiste, qui présente de réels dangers pour l'âme généreuse. Comment ne pas replacer ce strip dans son contexte? La critique se fait ensuite plus explicite dans Une bouteille à la mer (Flaschenpost, 27/1937), où l'auteur raille l'esprit allemand. Le père envoie un message dans une bouteille pour excuser l'absence de son fils. Or, la réponse du directeur d'école, preuve que le message est bien arrivé et que le directeur sait que père et fils sont en danger, atteint quelques temps plus tard les deux Robinson: le directeur demande des preuves de l'allégation du père selon laquelle lui et son fils sont portés disparus...

L'analyse de plusieurs saynètes de Vater und Sohn indique qu'il est tout à fait possible, compte tenu des circonstances, de voir dans cette œuvre une réflexion sur l'humain mais aussi sur une époque, entre rire et larmes. Au fil des événements et de l'évolution de l'auteur, la critique se fait à la fois plus fine et plus présente. De toute évidence, la bande dessinée s'est présentée pour e.o.plauen comme un medium souple et efficace pour poursuivre sa création artistique et partager ses vues sur la vie et son époque. Nous examinerons donc maintenant en quoi l'auteur a su exploiter les ressources spécifiques de la BD pour obtenir ce résultat.

\section{Exploiter les ressources de la BD pour contourner la censure}

La BD est un support d'expression autonome, elle offre à ce titre à l'auteur certaines possibilités qui lui sont spécifiques. Ce sont ces ressources que nous nous proposons d'étudier maintenant. Nous nous concentrerons dans un premier temps sur les contraintes éditoriales avant de voir comment l'auteur joue avec les contraintes du scénario puis du dessin pour se créer un espace de liberté créative. Nous nous interrogerons, dans un dernier temps, sur l'utilisation extrêmement parcimonieuse qu'il fait des légendes, dans un contexte où le langage est dévoyé, comme l'a montré Otto Klemperer (1996).

Rappelons que la BD est d'abord née du feuilleton: répondant à une dynamique éditoriale, principalement américaine, elle se présenta tout d'abord sous la forme de strips réguliers, quotidiens ou hebdomadaires, de quelques vignettes à une ou plusieurs pages (A. Baron-Carvais, 2007). Elle mettait en scène des personnages constants, dans 
un cadre diégétique lui aussi fixé. Les aventures des personnages se poursuivaient de jour en jour sur le principe du feuilleton à rebondissements (Ce fut le cas, après guerre, des Aventures de Blake et Mortimer, par exemple) ou bien sous la forme de saynètes indépendantes, chaque gag formant un tout cohérent. Les strips peuvent alors suivre un fil rouge, constituer une histoire ou bien être réellement indépendants les uns des autres, comme c'est le cas pour Vater und Sohn.

Les premières contraintes que dut respecter e.o.plauen furent donc celles du sujet et de la livraison hebdomadaire. Il était en effet tenu de mettre en scène des personnages constants dont les aventures puissent quelque peu égayer le quotidien des lecteurs. En outre, chaque semaine, il devait présenter plusieurs planches et l'éditeur choisissait celles qu'il jugeait publiables. Ce cadre n'offrait évidemment qu'un espace extrêmement restreint de contestation et pourtant, l'auteur a su en jouer et créer un nouveau monde.

Le premier point, le plus évident, explicite dans le titre de la BD, est la relation extrêmement étroite, tour à tour complice, tendue ou marquée du sceau de l'autorité, entre père et fils. La mère n'apparaît qu'une fois (La fabuleuse lecture de Noël, Das interessante Weihnachtsbuch, 51/1934). Le scénario imposait un troisième personnage mais la dynamique est tout à fait univoque : la mère demande au père d'aller chercher son fils, que l'appel du ventre n'arrache pas à sa fascinante lecture. Ne voyant personne revenir, elle finit par aller voir ce qui se passe et trouve les deux hommes plongés dans la lecture dont le premier devait sortir le second. En somme, la mère est seulement là pour les besoins de la pièce, mais père et fils forment une unité imperméable. La seule autre femme qui intervienne dans la relation père-fils est la sirène (cf. plus haut), qui, de toute manière, disparaît et laisse père et fils dans une commune tristesse. La proximité entre père et fils est le fil conducteur de toute la série : la relation entre ces deux être atypiques, très éloignés des idéaux propagés par le Troisième Reich, forme un univers clos et rassurant. Le père ventripotent et le fils rebelle, voire un brin anarchiste, ne répondent guère à l'idée de l'homme aryen, grand et athlétique, uniforme et tout entier dédié à la communauté, dans laquelle il se fond. En quelque sorte, ces deux personnages tendaient aux Allemands de l'époque, qui ne respectaient pas tous, et de loin, les idéaux du régime, un miroir rassurant de leur normalité. Sous couvert de présenter une relation aussi attachante que banale, l'auteur ouvre donc discrètement les portes de la contestation en suscitant une réflexion de ses lecteurs sur les normes du régime.

Or, les aventures de ces deux personnages si peu conformes à l'idéal de la Volksgemeinschaft évoluent. L'auteur se les approprie et peut progressivement introduire des pointes amères ou grinçantes sans éveiller la méfiance d'une censure routinière ou d'un lecteur peu attentif, qui ne verraient dans un gag qu'une innocente et attendrissante histoire entre un père et son fils. L'artiste, dans un premier temps, maitrise ses personnages puis il les fait évoluer sur la longue durée et évolue avec eux. Ceci est encore plus évident dans le cas de la BD, à livraison hebdomadaire, que dans celui du roman ou du théâtre, par exemple. E.o.plauen a grandi avec ses personnages et a façonné leur caractère pendant trois ans, semaine après semaine. Il paraît en ce sens tout à fait légitime de supposer qu'il s'est progressivement détaché des contraintes qui le cantonnaient dans la BD innocente pour trouver sa voie (ou sa voix, en un sens) et retrouver, dans le cadre imposé, le ton férocement critique qui était le sien dans la caricature. Cela apparait clairement dans deux bandes publiées, respectivement, en 
1938 et, à titre posthume, en 1953. Dans la première, En deuil de la femme sciée en deux (Trauer um die zersägte Dame, publication dans le troisième recueil édité par Ullstein, 1938), le père et le fils, assistant à un numéro de magie, croient sincèrement que l'assistante du magicien est sciée en deux. Quel n'est pas leur étonnement, quand ils reviennent avec une couronne funéraire, de retrouver la même femme sur scène !
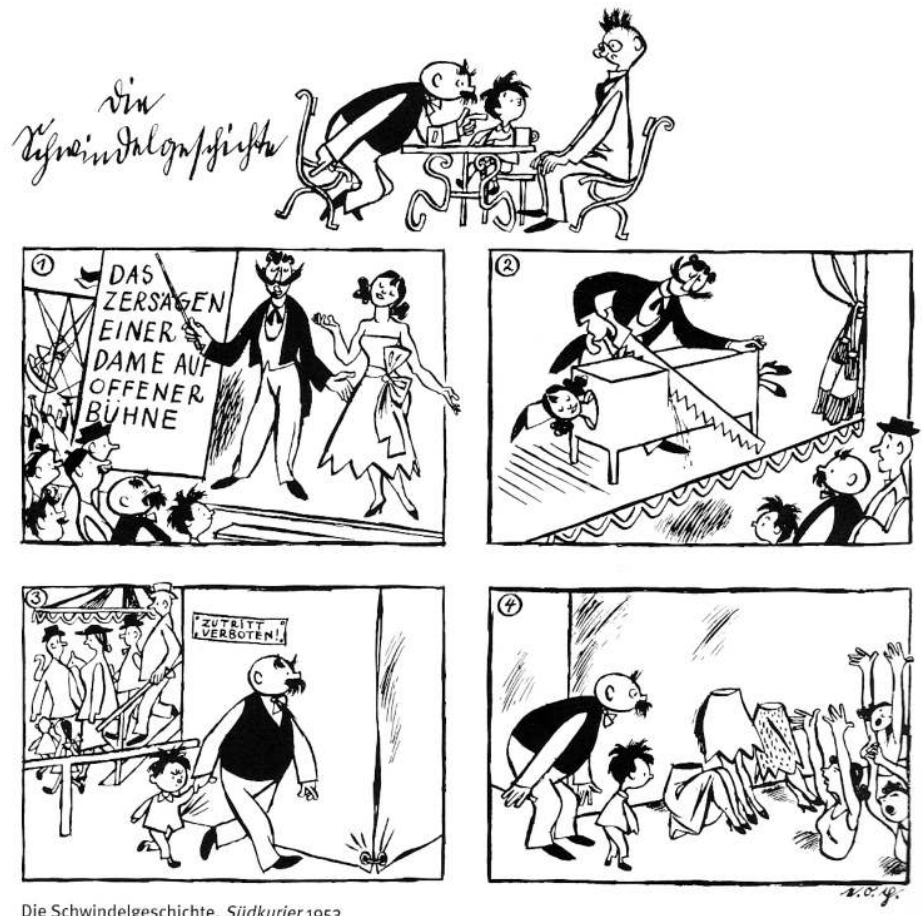

Die Schwindelgeschichte, Südkurier 1953

Source : Ohser, E. (e.o.plauen), Gesamtausgabe, Politische Karikaturen, Zeichnungen, Illustrationen und alle Bildgeschichten Vater und Sohn, Contance, Südverlag GmbH, 2000.

Dans le deuxième strip, paru dans la Südkurier, L'escroquerie (Schwindelgeschichte), père et fils, ayant assisté au même numéro, vont en coulisse et trouvent des parties de femmes réellement sciées ${ }^{3}$. Or, ce thème avait fait l'objet d'une caricature de 1932 (Surprise derrière le stand!, Zauber hinter der Bude) ou encore dans Les gardiennes de la morale font congrès (Die Tagung der Sittlichen, Querschnitt, 1930: des militantes bourgeoises et dépourvues de corps à partir du bas-ventre, écoutent avec conviction une conférencière amputée comme elles). À l'inverse, en 1935, dans Les phoques (Besuch bei den Robben, 3/1935), e.o.plauen mettait en scène père et fils au zoo, où le fils, au grand mécontentement de son père, notait la ressemblance entre les moustaches des phoques et celles de son père; en 1939, dans Surprise au zoo (Überraschung im Zoo, Berliner Illustrirte, 1939) il caricaturait une femme de la bonne bourgeoisie dont le haut chapeau cylindrique attire les rhinocéros unicornes, qui semblent voir en elle une parente. Le gag plutôt innocent se transforme en critique sociale, alors que, dans le cas précédent, la critique du début prenait une forme plus édulcorée mais non moins dérangeante dans la BD. Le dessinateur est resté fidèle à son caractère, à son style et à ses idées et poursuit prudemment sa critique. Si, comparées aux caricatures parues dans le Vorwärts, les premières histoires de Vater und Sohn apparaissent comme une évidente volte-face, elles ne le restent pas et l'auteur apprend à jouer avec les contraintes éditoriales et la censure pour transmettre ses pensées sans s'exposer 
inutilement... à tel point, peut-être, que la critique n'est plus perceptible sans effort particulier.

Un autre jeu spécifique à la BD est celui de la fiction. Selon P. Fresnault-Deruelle, « de même que les univers de Bosch, Bruegel, Moreau, Ernst, etc..., multiplient à l'infini le paradigme du cheval ailé, de même nos nouveaux graphistes (les cartoonistes), en une déviance quasi perverse, déplacent les références spatio-temporelles et biologiques auxquelles nous sommes habitués » $(1977,105)$. La BD prend en somme son lecteur par la main dans son parcours imaginaire et rend très vraisemblable ce qui est plutôt invraisemblable, voire impossible.

Parmi les exemples que nous avons commentés, certains reposent clairement sur une fiction qui se donne comme réalité : la baleine dans la baignoire qui finit par faire exploser la maison, le chien qui va chercher et ramène la balle du fusil... D'autres cas sont plus retors parce que l'entrave aux lois de la vraisemblance est moins évidente: ainsi de La peinture a ses limites (Grenzen der Malerei, 6/1935) : le fils, ayant cassé la glace murale, déblaie les bris de glace et peint son père avec un nœud papillon. Le père fait son nœud de cravate devant le supposé miroir avant de s'apercevoir, stupéfait, que son reflet porte un nœud papillon. Situation complètement invraisemblable mais qui ne choque pas au premier abord comme telle. Avant tout, la liberté prise avec le réel permet la métaphore et elle élève aussi le gag à un niveau symbolique, qui fête avant tout l'humanité des deux héros dans leurs bons comme dans leurs mauvais côtés. L'auteur pose ainsi des valeurs morales d'humanité sans jugement. C'est au lecteur d'estimer le décalage entre ce monde, qui pourrait être le sien si le cours de l'histoire en décidait autrement, et le quotidien du Troisième Reich. La critique sous-jacente du régime par contraste avec le monde fictif $\mathrm{du}$ père et $\mathrm{du}$ fils fonctionne finalement d'autant mieux que le monde des deux héros est comparable (mais aussi différent) à celui des lecteurs.

Un dernier point essentiel et qui pourrait faire l'objet d'une étude à part est celui de l'utilisation des légendes et de sa signification dans le contexte de Vater und Sohn. Les paroles brillent avant tout par leur rareté. Cela rend leurs rares apparitions d'autant plus remarquables. Les paroles apparaissent quasiment systématiquement dans des situations absurdes et sont reliées à la duperie, à la violence ou à la réprobation. Ainsi, dans Le Courrier des poissons, le fils, refusant de sacrifier les poissons au plaisir de la pêche, écrit une lettre qu'il accroche à l'hameçon de la ligne paternelle, et le père peut alors lire le message des poissons qui ne sont pas disposés ce jour-ci (Der Brief der Fische, 20/1935). Le fils arrive de même à dérober aux yeux et à la barbe de son père un pot de confiseries qu'il va manger dans sa chambre en jouant le somnambule. Sur une pancarte accrochée à son cou, "Somnambule, ne pas réveiller, danger de mort " (Le Somnambule, Der Schlafwandler, 33/1935). Les paroles suivent la même évolution que le reste de la $\mathrm{BD}$ et les messages deviennent plus amers avec le temps. Ainsi, dans L'Éducation par la pratique (Praktische Erziehung, 20/1937), le père lit des passages d'un livre à son fils. Son fils, peut intéressé, bricole et finit, dans son jeu, par lancer une pomme à la figure de son père, qui lance son livre sur lui. On lit alors le titre : «Éduquer sans châtier» (Erziehung ohne Züchtigung). L'ironie se fait encore plus amère avec la bouteille à la mer (exemple commenté plus haut), où le père et le fils, naufragés, se voient sommés par le directeur d'école de justifier l'absence du fils... Au demeurant, le langage n'a guère joué son rôle de moyen de communication. Que dire encore du cas où le père et le fils lancent dans l'eau une bouteille avec une lettre exposant leur désir 
d'échange ? La bouteille vient frapper un nageur, qui, en guise d'échange, poursuit le père et le fils en brandissant la coupable bouteille (Un vœu accompli sans délai, Schnell erfüllter Wunsch, 33/1936). De manière significative, les mots les plus sereins sont les mots d'adieu dans le dernier strip, "Chers amis, au revoir, Père et fils » (Adieu, la plus grande aventure, Abschied, das größte Abenteuer, 49/1937).

L'usage que fait l'auteur du langage, d'ailleurs écrit et jamais oral, si l'on excepte quatre exceptions, dont l'une est une interjection d'un perroquet et les trois autres de simples interjections (Prosit Neujahr, bonne année, Siehste?, vois-tu? et Tor!, But!), indique le désir de rester dans le domaine du ressenti, de l'expérience vécue et des sentiments non verbalisables. Le langage apparait disruptif, il introduit la discorde, le désagrément, la colère ou l'absurdité, le plus souvent. Ceci, dans le contexte du Troisième Reich, n'est pas anodin. V. Klemperer écrivait ainsi, au sujet de l'abréviation, dans La langue du Troisième Reich: "Aucun style de langage d'une époque antérieure ne fait un usage aussi exorbitant de ce procédé que l'allemand hitlérien. [...] Le nazisme technicise et organise justement tout. [...]. Il tente aussi, au nom de cette exigence de totalité, de s'emparer de toute la vie intérieure $[. .$.$] » (1996,130)$. Nous faisons donc l'hypothèse que l'auteur a sciemment renoncé à la parole pour insister sur la force des liens au-delà du langage, non verbalisables. Cela décuple la force des sentiments et permet à e.o.plauen, avec le recours au rapport assez libre au vraisemblable, comme nous venons de le voir, de créer un monde véritablement à part et qui, par sa seule existence, est en soi une critique de l'ordre imposé par le Troisième Reich. La critique, avec le temps et l'expérience de l'auteur, se fait du reste toujours plus explicite, même si elle ne dépasse jamais la ligne rouge qui aurait signifié l'interdition par la censure (faute d'archives, du reste, nous ne pouvons pas non plus exclure que e.o.plauen se soit autorisé des strips plus explicites au sujet du régime mais qui n'auraient pas été publiés).

\section{La censure n'est plus... l'ingratitude de la postérité}

Vater und Sohn, loin d'être un gentil divertissement, livre une vision du monde qui, mise en perspective, porte une critique de la dictature. Il serait injuste de dénier à Erich Ohser, qui a payé de sa vie sa dissidence, le courage de la critique. Il est au contraire essentiel de rechercher dansVater und Sohn les traces de la contestation.

Pour cela, il est avant tout nécessaire de se départir de l'opinion couramment répandue selon laquelle la BD serait un art apolitique, plutôt adressé à un public jeune et indigne d'un public cultivé et politisé. Vater und Sohn connut un succès retentissant parce qu'elle donnait à voir à chacun ce qu'il voulait voir. La méprise dans la réception de Vater und Sohn est ironiquement la meilleure preuve que cette BD possède toute l'ambiguïté que l'on peut attendre d'une oeuvre née sous la censure : elle transmet son message, critique sans concession, mais avec un tel art de la dissimulation que le lecteur inattentif ou prévenu, comme la censure en son temps, reste à la surface et vide l'œuvre de sa véritable dimension artistique. 


\section{BIBLIOGRAPHIE}

Baron-Carvais, A., La bande dessinée, Paris, PUF, Collection Que Sais-je, 2007.

Fresnault-Deruelle, P., La bande dessinée, Paris, A. Colin, 2009.

Fresnault-Deruelle, P., Récits et discours par la bande, Essai sur les Comics, Paris, Hachette, 1977.

Klemperer, V., LTI, La langue du IIIe Reich, Carnets d'un philologue, Paris, A. Michel, (Trad :

E. Combe), 1996.

Klinkers, F., Der Zeichner Erich Ohser (1903-1944), Thèse de doctorat, Berlin, 1976.

Laubach, D., « Erich Ohser : Leben und Schaffen », in Ohser, E. (e.o.plauen), Gesamtausgabe, Politische Karikaturen, Zeichnungen, Illustrationen und alle Bildgeschichten Vater und Sohn, Konstanz, Südverlag $\mathrm{GmbH}, 2000$, pp. 11-33.

Möller, H., Exodus der Kultur, Schriftsteller, Wissenschaftler und Künstler in der Emigration nach 1933, Munich, C. H. Beck, 1984.

Ohser, E. (e.o.plauen), Gesamtausgabe, Politische Karikaturen, Zeichnungen, Illustrationen und alle Bildgeschichten Vater und Sohn, Contance, Südverlag GmbH, 2000.

Reichel, P., Der schöne Schein des Dritten Reiches : Faszination und Gewalt des Faschismus, Frankfurt am Main, Fischer Taschensbuch, 1993.

Ueding, G., « Ungeheuer aus der Tiefe der politische Zeichner Erich Ohser », In : Wirkus, B. (éd.), Die kulturelle Moderne zwischen Demokratie und Diktatur. Die Weimarer Republik und danach, Constance, UKV GmbH, 2007, pp. 335-344.

\section{NOTES}

1. «Rares furent les publications qui s'offraient aux lecteurs comme des critiques cryptées du régime, que telle fût leur intention ou non ».

2. „Flucht in die Idylle oder in die sogenannten einfachen und zeitlos menschlichen Verhältnisse, Flucht in den Traditionalismus, in die forcierte Betonung des alten Wahren und Unvergänglichen, Flucht in das Bewährte und damit Problemlose, Flucht nicht zuletzt vor der Trivialität und der Barbarei in das Schöne, Edle und Ewige.“

3. La date de publication ne renseigne malheureusement pas sur la date de création, nous supposons donc que ce sont des dessins tardifs, comme le laisse entendre l'évolution des strips effectivement datés.

\section{RÉSUMÉS}

Vater und Sohn, la BD de e.o.plauen publiée sous le Troisième Reich entre 1934 et 1936, connut un immense succès. Le père et le fils sont devenus de véritables figures, que l'on retrouvait aussi 
bien sur des billets de loterie que sous forme de jouets divers ou encore comme animateurs lors de manifestations, par exemple d'œuvres caritatives, une œuvre que l'on associerait plus à la veine commerciale de Walt Disney qu'à l'univers du Troisième Reich. Un monde féérique sous le Troisième Reich ? À première vue, on pourrait prétendre que Vater und Sohn est une œuvre sympathique et pleine d'humour qui devait représenter, dans un contexte oppressant, une oasis d'humanité et de bonne humeur, un moyen d'évasion fort efficace. Mais il est aussi possible, comme nous nous proposons de le faire, de chercher, au-delà du vernis d'harmonie et de sérénité, un message crypté de l'auteur, qui laisse percevoir l'inquiétude et les angoisses d'un caricaturiste de la République de Weimar réduit, pour gagner sa vie, à dessiner sous l'autorisation et la censure du Troisième Reich. La question est alors de savoir comment l'auteur a utilisé les ressources propres de la BD pour distiller prudemment ses doutes, ses peurs, ses angoisses sans s'exposer à la censure. Et aussi de savoir si la prudence de mise de l'auteur ne compte pas pour beaucoup dans la réception de l'œuvre comme BD innocente et sans prétention critique ?

E.o.plauens zwischen 1934 und 1936 erschienener Comic Vater und Sohn erzielte einen durchschlagenden Erfolg. Vater und Sohn sind zu echten Figuren geworden und man konnte Ihnen teilweise auf Lotteriescheinen, als Spielwaren oder noch als Animateuren bei Wohltätigkeitsveranstaltungen begegnen. Dies gemahnt eigentlich eher an eine erfolgreiche Vermarktung à la Walt Disney als an die Atmosphäre des Dritten Reiches. Eine Märchenwelt im Dritten Reich? Vater und Sohn erscheint nämlich beim ersten Anblick als ein humorvolles und gemütliches Werk, das im Dritten Reich zweifellos als eine Quelle der Freude und der Menschlichkeit gegolten haben muss. Die Flucht aus dem grauen Alltag schlechthin. Dafür kann das Werk auch anders betrachtet werden : ein Blick hinter die Kulisse vermittelt einen anderen Eindruck. Statt Harmonie und Glückseligkeit lässt sich eine verschlüsselte Botschaft wahrnehmen, eine Botschaft von den Ängsten und dem Unbehagen eines Karikaturisten der Weimarer Republik, der dazu gezwungen wurde, sich unter die Knute der Zensur zu ducken, um seinen Lebensunterhalt zu verdienen. Wie konnte der Autor die Mittel, die ihm der Comic bot, dazu benutzen, um seine Zweifel und Ängste vorsichtig genug zum Ausdruck zu bringen, ohne sich in den Augen seiner Zensoren strafbar zu machen? Ist die gebotene Vorsicht des Künstlers nicht etwa für die Verharmlosung des Werkes verantwortlich?

E.o.plauens' comic strip Vater und Sohn, published between 1934 and 1936, was an immense success. Father and son quickly became popular characters, which could be found on lottery tickets, or as toys and even as entertainers at charity performances. It was a commercial success story worthy Walt Disney rather than the Third Reich. At first sight, Vater und Sohn may appear as a pleasant and witty piece of visual art full of humour and humanity, enabling the reader to effectively escape from the oppressing reality of the period. Another reading of the comic strip is however possible. Behind the images of good-natured harmony, a concealed message is to be perceived - it reveals the fears and discomfort of a cartoonist of the Republic of Weimar, who had no choice but to comply to censorship to make a living. In this article, I analyze how the author uses the means offered by the comic strip to express his fears and doubts without arousing the censors' suspicions. The caution the author had to exercise may largely explain why the comic strip has been perceived as innocent and without critical value.

\section{AUTEUR}

\section{SYLVAIN FARGE}

Université Lumière Lyon 2 